\title{
A Data-Driven Sensitivity Analysis Approach for Dynamically Positioned Vessels
}

\author{
$\mathrm{Xu}$ Cheng ${ }^{1,2}$ Robert Skulstad ${ }^{2}$ Guoyuan $\mathrm{Li}^{2}$ Shengyong Chen ${ }^{1}$ Hans Petter Hildre ${ }^{2}$ Houxiang \\ Zhang $^{2}$ \\ ${ }^{1}$ School of Computer Science and Technology, Tianjin University of Technology, China \\ chengxutjutegmail.com, csyetjut.edu.cn \\ ${ }^{2}$ Department of Ocean Operations and Civil Engineering, Norwegian University of Science and Technology, Norway \\ $\{x u . c h e n g$, robert.skulstad, guoyuan.li, hans.p.hildre, hozh $\}$ a nt nu. no
}

\begin{abstract}
For safety-critical marine operations, the dynamically positioned (DP) vessel should maintain a predetermined heading and position for varying environmental conditions using the thrusters. Studying the effect of each thruster to the capability of DP vessels is significance but challenging. This paper presents a data-driven and variance-based sensitivity analysis (SA) approach that can dig into the ship sensor data to estimate the influence of each thruster for DP operations. Considering high-computational cost of variance-based SA, an Extreme Learning Machine (ELM) -based SA is proposed. To apply the SA to sensor data, an ANN is built and trained on the basis of ship sensor data and then employed as a surrogate model to generate Monte Carlo (MC) samples. A benchmark test shows the correctness of the proposed approach. A case study of SA in DP operation is conducted and the experimental results show that the proposed approach can rank and identify the most sensitive factors. The proposed approach highlights the application of variance-based SA in data-driven modeling for ship intelligence.
\end{abstract}

Keywords: dynamical positioning, sensitivity analysis, thrust analysis, data-driven modeling

\section{Introduction}

With the development of oil and gas exploration and other operations in the deep sea, DP vessels have obtained more and more attention. A DP vessel is defined as a vessel that can maintain a specified position and heading automatically by employing its thrusters to guarantee the continuous offshore operation (Sørensen, 2011). The safety of operation of the DP vessel is often the first consideration to be paid attention to, especially in those safety-critical operations such as drilling, oil production, and off-loading, where the positioning and heading accuracy are very high, regardless of the environmental conditions. To satisfy the critical requirements, it is necessary to understand the effect of each thruster to against the environmental factors (Xu et al., 2015; Pivano et al., 2012).

However, it is not an easy task to study the influence of each thruster in DP vessels. Mahfouz and El-Tahan de- veloped a software program with the aim of selecting and configuring the thrusters for the newly designed DP vessels (Mahfouz and El-Tahan, 2006). A program which is named as DPCAP is developed by the Maritime Research Institute Netherlands (MARIN) with the purpose of analyzing the thruster capacity of the DP vessels. The main drawback of the DPCAP is that there is no quantitative method to compare the different thruster configurations. To improve the ability of DPCAP, Xu et al. proposed a novel thrust sensitivity analysis on the basis of a newly defined synthesized positioning capability criterion for marine vessels (Xu et al., 2015). The main limitation of this method is that it employs local sensitivity analysis (LSA), which can only reflect the characteristics of the synthesized capability at some fixed points in the input space.

To address the limitation of Xu's method to study the effect of each thruster in DP vessels, a data-driven and variance-based SA approach is proposed. The approach utilizes the historical data of DP to analyze the influence of each thruster. In addition, in order to be able to apply the proposed method to most marine operations to mine useful information, this paper also proposes a framework for data analysis in marine operations.

Our on-going project aims to develop intelligent systems to support decision making in various maritime operations. A new integrated platform including data analysis tools, and data-driven modeling technique is designed, which will serve the maritime industry for improving operational effectiveness and safety. In this paper, only data analysis tools part would be focused on. The variancebased SA would be employed to understand the DP data (Fernández-Navarro et al., 2017). In the case of only sensor data available, it is still a challenge to employ the variance-based SA directly to the sensor data of the DP vessels. Thus, a fitted and validated surrogate model representing the motion of DP vessel is constructed on the basis of sensor data that are observed in a simulated vessel. To accelerate the SA, an ELM is adapted. The contribution of this paper includes (1) introducing a framework of data analysis for offshore operations, and (2) providing a data-driven SA method that is capable of coping with the SA on the DP data. 


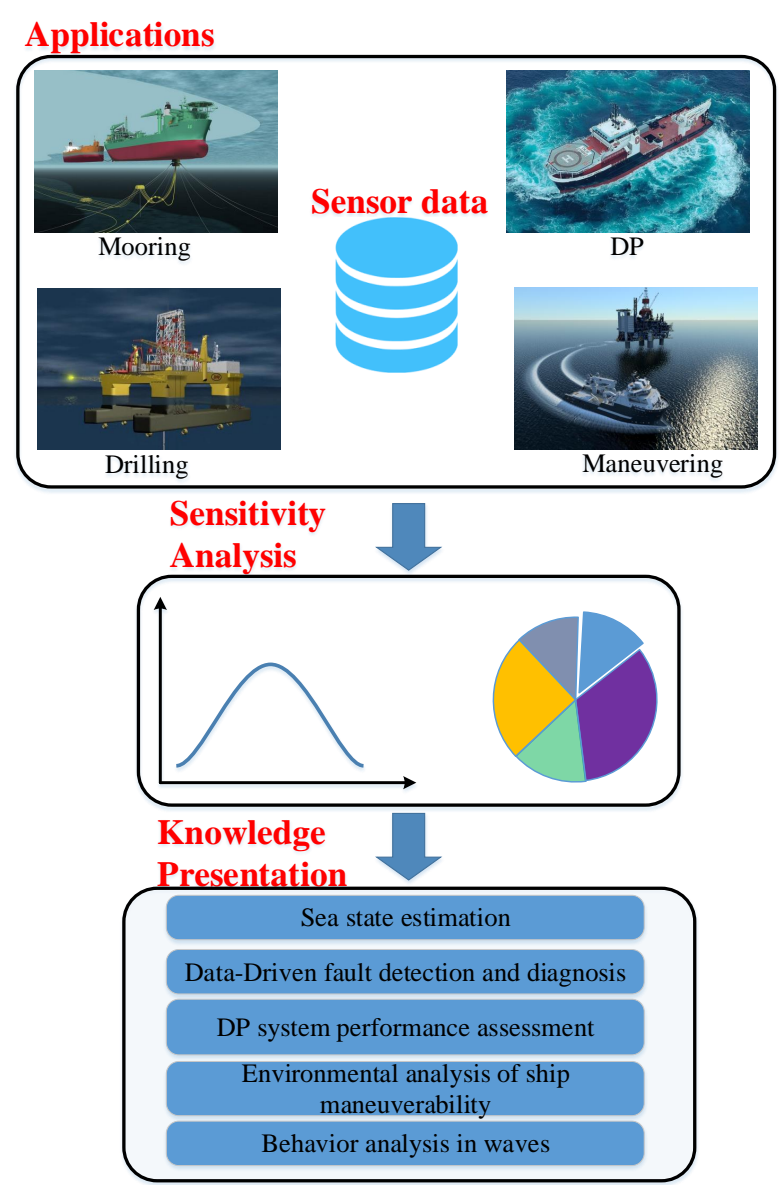

Figure 1. Framework of data analysis in offshore operations.

The rest of the paper is organized as follows. Section 2 shortly introduces the overall structure of the data analysis framework. In Section 3, the ELM-based sensitivity analysis is described in detail. Section 4 presents case studies and evaluation results. Conclusion and future work are shown in Section 5.

\section{Framework of data analysis for off- shore operations}

Due to the significant uncertainties and various operating conditions, offshore operations are complex and hazardous. Operational safety is a major issue and is easily challenged by harsh marine environments, complex geological conditions, and human and equipment factors. The conventional model-based solutions require an in-depth knowledge of the offshore operations, which are impractical for complicated offshore operations. Fortunately, with the rapid development of technologies such as data collection, data mining and artificial intelligence, the extraction of useful information has been significantly improved in the offshore operations ( $\mathrm{Li}$ et al., 2016, 2017). Hence, data-driven technology is an alternative that can be used for efficient operational monitoring and data analysis (Cheng et al., 2017; Wang et al., 2016).

A considerable amount of sensor data has been accumu- lated in all kinds of offshore operations. Analyzing these data has the following difficulties: 1) the sensor data to be analyzed are usually too large and high dimensional; 2) they usually contain measurement-induced noise and redundant information, which makes it difficult to analyze accurately; 3) It is not easy to intuitively interpret the data from multiple sensors. Furthermore, there are many uncertainties in these data, which are influenced by a variety of factors, such as weather conditions, human factors, and so on. How to characterize and reduce that uncertainty is becoming more and more popular in engineering research. As an aspect of uncertainty quantification, the SA is defined as the investigation of "how uncertainty in the output of a model (numerical or otherwise) can be apportioned to different sources of uncertainty in the model input factors" (Saltelli, 2002). SA has been widely used for industrial applications with different purposes, mainly including assessing the uncertainty, calibrating the model, and making robust decision (Pianosi et al., 2016). In general, SA could be implemented in either a local or a global manner. The difference is that the former analyzes the effect of a single input on the output and treats the other inputs as deterministic values; whereas the latter examines the sensitivity from the perspective of the entire range of each input's variation.

Figure 1 illustrates a possible scheme of the data analysis for offshore operations. Note that the sensitivity analysis module is the core of the scheme. It takes the sensor data from numerous offshore applications as input and the designated metric, e.g., the capability of DP vessels, as output, to quantify how much the input contributes to the output. The result can benefit both the DP system performance assessment and behavior analysis in waves. For example, if one thruster accounts for one of the main factors for DP system, this element will be given more attention, since, if it fails, the remaining thrusters may not possible produce sufficient forces to keep the vessel on the predetermined position and heading. The information from the sensitivity analysis can also be obtained to establish an estimator for sea state identification. Based on the framework, it is possible to implement an intelligent system that utilizes data analysis method, artificial intelligent algorithms and advance control theory to achieve ship intelligence for various purposes ranging from sea state estimation, risk assessment, manoeuvring evaluation, sensor diagnosis to behavior analysis.

\section{Proposed Approach}

To test the feasibility of the introduced data analysis framework, a data-driven sensitivity analysis approach for DP operations is proposed, as shown in Figure 2. The process of the proposed approach is as follows: (1) Training the artificial neural network (ANN) to generate a surrogate model on the basis of DP sensor data. (2) Validating the model. (3) Performing variance-based SA on the well-trained surrogate model. In order to accelerate 


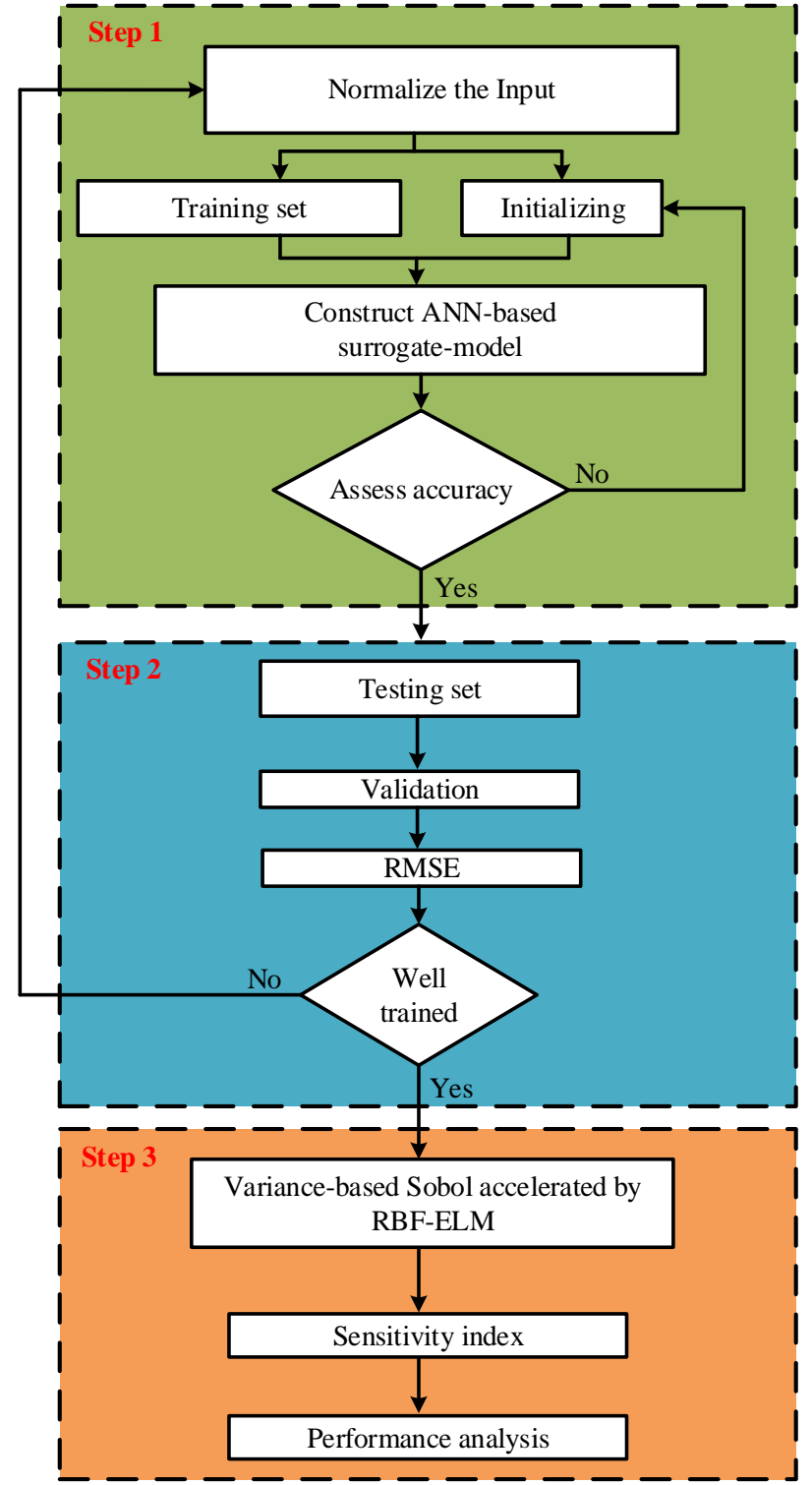

Figure 2. Workflow of the proposed approach.

Sobol' method, Radial basis function (RBF)-based ELM is integrated into Sobol.

\subsection{ANN-based surrogate model}

The data-driven ANN is an essential part of the scheme. It has multiple roles: First, it provides a bridge between the modeler and the sensor data to better simulate and understand the ship's behavior. Second, the data-driven ANN model is employed to generate a number of input parameters using Latin Hyper-cube Sampling (LHS) technique and to calculate the corresponding output. In this paper, a three-layer feed-forward neural network is established for the proposed method. The Back-Propagation (BP) algorithm is used as a learning algorithm. Sigmoid functions and linear functions are used as activation functions for the hidden and output layers, respectively. During the training phase, $80 \%$ of sensor data is used for training, and the remaining $20 \%$ is used for testing and validation.

\subsection{ELM-Sobol SA method}

Assuming the model form is $f(\boldsymbol{X})=f\left(x_{1}, \ldots, x_{M}\right)$, where $\boldsymbol{X}=\left(x_{1}, \ldots, x_{M}\right)$ represents the model input which contains $M$ independent parameters. The model output can be decomposed as follows (Saltelli and Sobol', 1995; Li et al., 2006):

$$
f(\boldsymbol{X})=f_{0}+\sum_{i=1}^{M} f_{i}\left(x_{i}\right)+\sum_{1 \leq i \leq j \leq M} f_{i j}\left(x_{i}, x_{j}\right) .
$$

Eq.(1) is known as ANOVA-representation (Analysis of Variance). $f_{0}$ is the mean of output. $f_{i}$ and $f_{i j}$ are the firstand second-order decomposition of output, respectively.

The variance of the model output can be obtained as follows:

$$
V_{y}=E\left(f(\boldsymbol{X})^{2}\right)-f_{0}^{2}
$$

The main sensitivity index can be defined as follows:

$$
S_{i}=\frac{V_{i}}{V_{y}}=\frac{V\left(E\left(f(\boldsymbol{X}) \mid x_{i}\right)\right)}{V_{y}}
$$

The Sobol' method employs the MC methods to calculate the sensitivity index which brings high computational complexity. In this paper, the idea of Wu et al. (Wu et al., 2016) is adapted to reduce the computational complexity of Sobol'.

Assuming the model output can be represented by the RBF-based ELM, which formulation is shown as follows:

$$
f(\boldsymbol{X})=\sum_{i=1}^{N} \omega_{i} \varphi_{i}(\boldsymbol{X})
$$

where $\varphi_{i}$ is the $i$-th basis function and $\omega_{i}$ is the coefficient of $i$-th basis function. According to Eq.(1), $f_{0}$ is the expectation of the model output.

$$
f_{0}=\int_{0}^{1} f(\boldsymbol{x}) d \boldsymbol{x}=\sum_{i=1}^{N} \int_{0}^{1} \omega_{i} \varphi_{i}(\boldsymbol{x}) d \boldsymbol{x}=\sum_{i=1}^{N} \omega_{i} \prod_{j=1}^{m} \psi_{i}^{j}
$$

The $\psi_{i}^{j}$ can be calculated using the Gaussian cumulative distribution function.

$$
\psi_{i}^{j}=c_{i} \sqrt{\pi}\left[\Phi\left\{\sqrt{2} \frac{1-x_{i}^{j}}{c_{i}}\right\}+\Phi\left\{\sqrt{2} \frac{x_{i}^{j}}{c_{i}}\right\}-1\right]
$$

where $\Phi$ is the Gaussian distribution function, and $c_{i}$ is the center of $i$-th basis function. The mean square of the model output can be computed as:

$$
\begin{aligned}
E\left(f(\boldsymbol{x})^{2}\right) & =\int_{0}^{1} f^{2}(\boldsymbol{x}) d \boldsymbol{x}=\sum_{k=1}^{N} \sum_{i=1}^{N} \int_{0}^{1} \omega_{k} \omega_{i} \varphi_{k}(\boldsymbol{x}) \varphi_{i}(\boldsymbol{x}) d \boldsymbol{x} \\
& =\sum_{k=1}^{N} \sum_{i=1}^{N} \omega_{k} \omega_{i} \prod_{j=1}^{m} \psi_{k i}^{j}
\end{aligned}
$$


where

$$
\psi_{k i}^{j}=e^{-\frac{x_{i}^{j}-x_{k}^{j}}{c_{i}^{2}+c_{k}^{2}}} c_{i k} \sqrt{\pi}\left[\Phi\left\{\sqrt{2} \frac{1-x_{i k}^{j}}{c_{i k}}\right\}+\Phi\left\{\sqrt{2} \frac{x_{i k}^{j}}{c_{i k}}\right\}-1\right]
$$

where $c_{i k}^{2}=\frac{c_{k}^{2} c_{i}^{2}}{c_{k}^{2}+c_{i}^{2}}, x_{i k}^{j}=\frac{c_{k}^{2} x_{i}^{j}+c_{i}^{2} x_{k}^{j}}{c_{k}^{2}+c_{i}^{2}}$

Considering the input factor $x_{d}$, the first order term is

$$
\begin{aligned}
f_{d}\left(x_{d}\right) & =E\left(y \mid x_{d}\right)-f_{0}=\int_{0}^{1} \int_{0}^{1} f(\boldsymbol{x}) \prod_{j=1, j \neq d}^{m} d x_{j}-f_{0} \\
& =\sum_{i=1}^{N} \omega_{i} \varphi_{i}\left(x_{d}\right) \prod_{j=1, j \neq d} \psi_{i}^{j}-f_{0}
\end{aligned}
$$

Then the partial variance of $x_{d}$ can be calculated as follows:

$$
\begin{aligned}
V\left[E\left(y \mid x_{d}\right)\right] & =E\left(f_{d}^{2}\left(x_{d}\right)\right)-f_{0}^{2} \\
& =\sum_{i=1}^{N} \sum_{k=1}^{N} \omega_{i} \omega_{k} \psi_{k i}^{d} \prod_{j=1, j \neq d} \psi_{i}^{j} \psi_{k}^{j}-f_{0}^{2}
\end{aligned}
$$

One can see that the Sobol' method can be computed by the coefficients of RBF.

\section{Experiment}

This section mainly refers to three relevant experiments which are used in verifying the feasibility of the method we proposed.

\subsection{Benchmark function}

The first subsection is dedicated to the validation of the proposed approach. The widely used benchmark Sobol' function (Kersaudy et al., 2015) in variance based GSA is employed to test the performance of the proposed approach. The expression of Sobol function is shown as follows:

$$
f(\boldsymbol{x})=\prod_{i=1}^{M} \frac{\left|4 x_{i}-2\right|+a_{i}}{1+a_{i}} \quad \boldsymbol{x} \in[0,1]^{M}
$$

In this paper, $M=7$ is the number of input parameters. The $a_{1}-a_{7}$ are set to $1,2,5,10,20,50,100$. First, the original samples are set big enough to generate a high accuracy ANN model, and 10 ANN models are created, and the best model is chosen from the 10 models based on the performance test using RMSE. The sensitivity results are listed in Table 1.

As the Table 1 shows, the proposed approach is com-

\begin{tabular}{|c|c|c|c|c|c|}
\hline \multirow[t]{2}{*}{ Input } & \multicolumn{3}{|c|}{$\begin{array}{l}\text { Samples of } \\
\text { Proposed approach }\end{array}$} & \multirow{2}{*}{$\begin{array}{l}\text { Samples of } \\
\text { Monte Carlo } \\
5000\end{array}$} & \multirow[t]{2}{*}{ Analytical } \\
\hline & 128 & 256 & 512 & & \\
\hline$x_{1}$ & 0.334 & 0.570 & 0.595 & 0.609 & 0.604 \\
\hline$x_{2}$ & 0.294 & 0.240 & 0.265 & 0.272 & 0.268 \\
\hline$x_{3}$ & 0.041 & 0.061 & 0.063 & 0.071 & 0.067 \\
\hline$x_{4}$ & 0.015 & 0.019 & 0.015 & 0.019 & 0.020 \\
\hline$x_{5}$ & 0.007 & 0.008 & 0.011 & 0.011 & 0.010 \\
\hline$x_{6}$ & 0.005 & 0.005 & 0.006 & 0.001 & 0 \\
\hline$x_{7}$ & 0.009 & 0.007 & 0.002 & 0.001 & 0 \\
\hline
\end{tabular}
petent to improve the computational efficiency but not sacrificing on precision, compared with the Monte Carlo method. The proposed approach significantly reduces the computational complexity regarding the number of ANN
Table 1. First-order sensitivity index of each input.

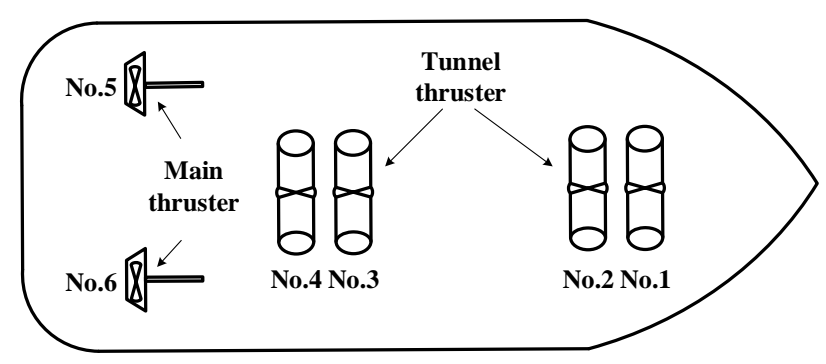

Figure 3. Thruster configuration

samples. Considering the complexity of a model, the proposed approach does not raise the computational complexity of conducting the variance-based GSA. Therefore, the more complex the model is, the more computation time would be reduced.

\subsection{Sensitivity result of the DP thrusters}

A sensitivity analysis for a DP vessel is conducted to assess how effectively the analysis determined the most sensitive thruster. The case ship model used is equipped with two tunnel thrusters in the bow, two tunnel thrusters and two main propellers at the stern, which is shown as Figure 3. The vessel is equipped with DP controller and the ship motion data and thruster data are collected.

For better analysis, we assume uniform, independent distributions for all parameters and initial conditions. The definition of input variability space then only requires defining the range of variation of each input factor. In this study, we chose to use ranges as wide as possible. Some parameters have the range according to the physical meaning of the parameters; the range of the others are confined by the minimum and maximum observed values of that variable over the entire data set.

As the performance of DP vessel is strongly related to the wind and wave, this section focuses on the influence of each thruster to the ship heading under different environmental effects. Therefore, ship heading was chosen as output variable, the output thrust of the six thrusters are considered as the input variables of the ANN model. The DP operation is simulated in the Offshore Simulator Centre AS (OSC), with the height of the wave assumed to remain constant at $2 \mathrm{~m}$ (ITTC spectrum and $15 \mathrm{~s}$ period). The wind increases from $4 \mathrm{~m} / \mathrm{s}$ to $12 \mathrm{~m} / \mathrm{s}$. Three scenarios with the wind and wave come from three different direc- 


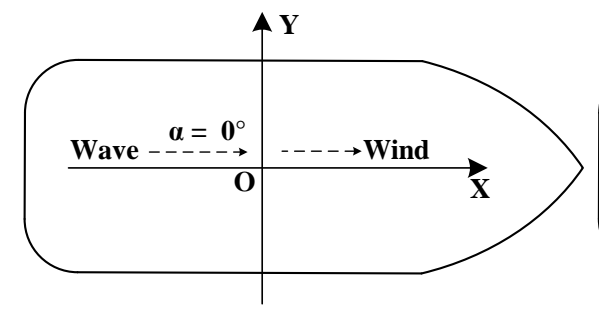

(a) Scenario 1

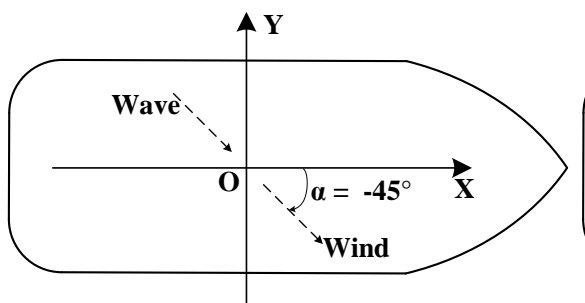

(b) Scenario 2 .

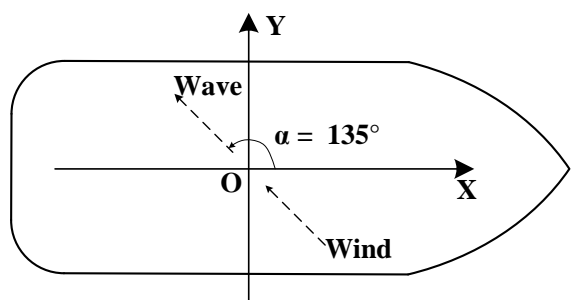

(c) Scenario 3

Figure 4. Three scenarios with different environmental factors.

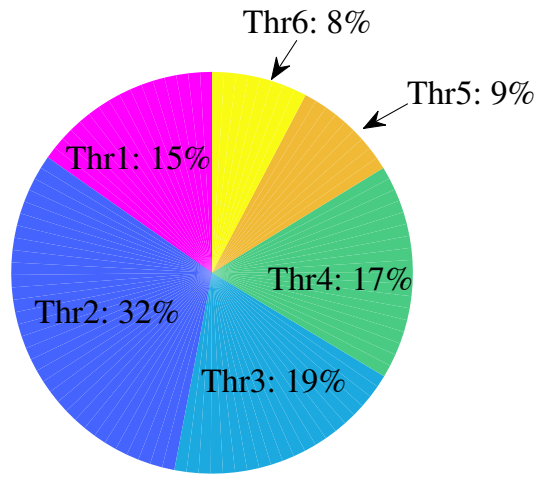

(a) Scenario 1 .

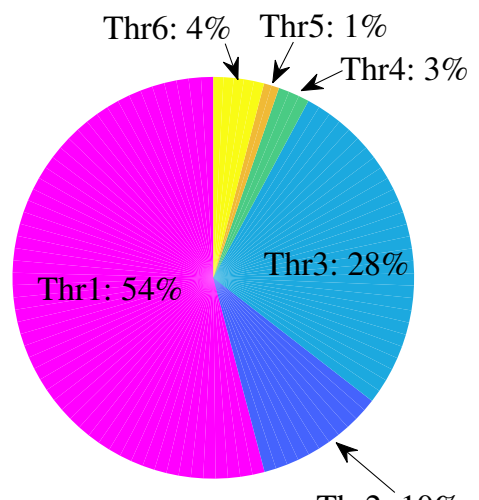

Thr2: $10 \%$

(b) Scenario 2

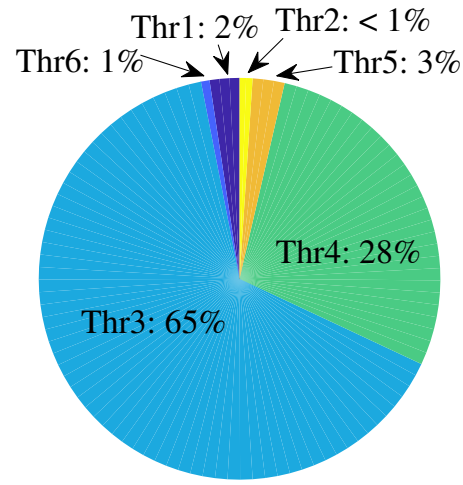

(c) Scenario 3

Figure 5. Sensitivity results of three scenarios.

tions are conducted, as shown in Figure 4.

Figure 5a, Figure $5 b$ and Figure $5 c$ show the sensitivity results of the three scenarios, respectively. In scenario 1 , it can be seen that there are four parameters (thruster 1 $\sim$ thruster 4 ) which are important to heading. The possible explanation for scenario 1 is that the transverse force and moment are highly depends on the four thrusters, and the wave and wind have no influence on the transverse force and moment. In scenario 2 , it is obvious that the most sensitive thruster is thruster 1 , and the thruster 3 follows. In order to overcome the transverse force generated by wind and waves, thruster 1 and thruster 3 supply sufficient forces in transverse direction. As showed in Figure $5 \mathrm{c}$, it is clear that the most sensitive thruster is the thruster 3. This is also recognized in the greater sensitivity result in thruster 4. As showed in Figure 4, the direction of wave and wind are the same. Hence, the expected thrust would be provided by those thrusters with orientation close to the direction of the environmental force. It can be concluded that, to improve the ability of DP in scenario 3. It is most effective to increase the propulsion capability of thruster 3.

\subsection{Knowledge understanding of DP}

In this paper, a dual-layer DP controller was used, including the motion controller and thruster force allocation controller. This paper mainly focuses on whether the thrust allocation is reasonable under different environmen- tal disturbances and whether it can maintain the ship's position and heading. When the wind and waves come from the longitudinal direction, the main thruster will generate strong thrust to counteract the influence of wind and waves. Note that the two main thrusters are operated using the same command and therefore does not contribute any torque about the transverse direction. In this case, the tunnel thruster with a large moment of force should have a greater influence on the heading angle. From the experimental results, as showed in Figure 5a, the thruster 2 has a large influence on the heading angle and the main thruster (thruster 5 and thruster 6 ) has relative lower influence. Therefore, the force allocation is reasonable under such circumstances. When the wind and waves come from the side, the thrust generated by the tunnel thruster near the environmental force should have a greater influence on the ship heading. The reason is that those thrusters close to environmental forces need to generate large thrusts to counteract environmental forces. From the experimental results of scenario 2 and scenario 3, when the force by the wind and wave is close to the orientation of thruster 1 and 2, the influence of thruster 1 and 2 should be greater. When the force by the wind and wave is close to the orientation of thrusters 3 and 4, thrusters 3 and 4 should have a higher influence. 


\section{Conclusion}

In this paper, a data analysis framework for offshore operations is introduced. An approach integrated ELM-based SA with ANN is proposed that can be applied for DP sensor data. This approach can be employed to quantify the influence of each thruster of DP vessels. To improve the capability of the DP vessels, the most effective way is to increase the thrust when the most sensitive thruster is identified. In order to verify the feasibility of this approach, a benchmark test is conducted. The benchmark test validated the feasibility of the proposed method used for conducting SA. Another experiment of SA on heading modeling in DP operation was conducted. The result shows that this approach can identify those influential factors that have an effect on the heading of the DP vessel. It is seen that the sensitivity analysis of thruster depends crucially on the environmental disturbances. Thus, the sensitivity analysis is of value in the preliminary design of the thrust system of DP vessels.

The sensitivity analysis of the thruster is highly timedependent. However, in this study, the surrogate model is ANN which cannot represent the dynamic of the DP vessel completely. Therefore, our future work will focus on investigating how to extend the proposed method to adapt to such external disturbances.

\section{Acknowledgement}

This research is partially supported by the project "SFI MOVE" funded by Norway Research Council, Norway (Project No: 237929). The authors would like to thank to the Offshore Simulator Centre AS for technical support. The author Xu Cheng would like to thank the sponsorship of the Chinese Scholarship Council for funding his research at Norwegian University of Science and Technology.

\section{References}

Offshore simulator centre. http://www. offsim. no/.

Xu Cheng, Shengyong Chen, Chen Diao, Mengna Liu, Guoyuan $\mathrm{Li}$, and Houxiang Zhang. Simplifying neural network based model for ship motion prediction: A comparative study of sensitivity analysis. In ASME 2017 36th International Conference on Ocean, Offshore and Arctic Engineering, pages V001T01A016-V001T01A016. American Society of Mechanical Engineers, 2017.

Francisco Fernández-Navarro, Mariano Carbonero-Ruz, David Becerra Alonso, and Mercedes Torres-Jiménez. Global sensitivity estimates for neural network classifiers. IEEE transactions on neural networks and learning systems, 28(11):2592-2604, 2017.

Pierric Kersaudy, Bruno Sudret, Nadège Varsier, Odile Picon, and Joe Wiart. A new surrogate modeling technique combining kriging and polynomial chaos expansions-application to uncertainty analysis in computational dosimetry. Journal of Computational Physics, 286:103-117, 2015.
Genyuan Li, Jishan Hu, Sheng Wei Wang, Panos G Georgopoulos, Jacqueline Schoendorf, and Herschel Rabitz. Random sampling-high dimensional model representation (rs-hdmr) and orthogonality of its different order component functions. The Journal of Physical Chemistry A, 110(7):2474-2485, 2006.

Guoyuan Li, Houxiang Zhang, Bikram Kawan, Hao Wang, Ottar L Osen, and Arne Styve. Analysis and modeling of sensor data for ship motion prediction. In OCEANS 2016-Shanghai, pages 1-7. IEEE, 2016.

Guoyuan Li, Bikram Kawan, Hao Wang, and Houxiang Zhang. Neural-network-based modelling and analysis for time series prediction of ship motion. Ship technology research, 64(1): 30-39, 2017.

Ayman B Mahfouz and Hussein W El-Tahan. On the use of the capability polar plots program for dynamic positioning systems for marine vessels. Ocean engineering, 33(8-9):10701089, 2006.

Francesca Pianosi, Keith Beven, Jim Freer, Jim W Hall, Jonathan Rougier, David B Stephenson, and Thorsten Wagener. Sensitivity analysis of environmental models: A systematic review with practical workflow. Environmental Modelling \& Software, 79:214-232, 2016.

Luca Pivano, Øyvind Notland Smogeli, and BjØrnar Vik. Dyncap-the next level dynamic dp capability analysis. Marine Cybernetics AS, 2012.

Andrea Saltelli. Sensitivity analysis for importance assessment. Risk analysis, 22(3):579-590, 2002.

Andrea Saltelli and Il'ya Meerovich Sobol'. Sensitivity analysis for nonlinear mathematical models: numerical experience. Matematicheskoe Modelirovanie, 7(11):16-28, 1995.

Asgeir J Sørensen. A survey of dynamic positioning control systems. Annual reviews in control, 35(1):123-136, 2011.

Hao Wang, Sindre Fossen, Fang Han, Ibrahim A Hameed, and Guoyuan Li. Towards data-driven identification and analysis of propeller ventilation. In OCEANS 2016-Shanghai, pages 1-6. IEEE, 2016.

Zeping Wu, Donghui Wang, Patrick Okolo, Fan Hu, and Weihua Zhang. Global sensitivity analysis using a gaussian radial basis function metamodel. Reliability Engineering \& System Safety, 154:171-179, 2016.

Shengwen $\mathrm{Xu}$, Xuefeng Wang, Lei Wang, Shuai Meng, and Bo Li. A thrust sensitivity analysis based on a synthesized positioning capability criterion in dpcap/dyncap analysis for marine vessels. Ocean Engineering, 108:164-172, 2015. 